the FISH based analysis to Nugent scoring, it may be inferred that FISH based analysis has an accuracy for the diagnosis of BV of 0.94 [95\% CI 0.86 - 0.98] (sensitivity 0.83 [95\% CI 0.51 - 0.97], specificity 0.97 [95\% CI $0.87-0.99$ ], PPV 0.83 [95\% CI $0.51-0.97]$, and NPV 0.97 [95\% CI 0.87 - 0.99].

Conclusion Urine-based diagnosis of bacterial vaginosis has a high accuracy. This method is particularly suited for epidemiological and clinical studies as fixated urine sediments can be stored for prolonged periods of time and the aliquots can be used for repeated FISH hybridisations under standardised conditions. In addition, urine samples are easily obtained in a non-invasive manner.

\section{P1.027 RISKS ASSOCIATED WITH BACTERIAL VAGINOSIS IN INFERTILITY PATIENTS: A SYSTEMATIC REVIEW AND META-ANALYSIS}

doi:10.1136/sextrans-2013-051184.0248

H Verstraelen, N van Oostrum, J Meys, P De Sutter. Ghent University, Ghent, Belgium

Background Several studies have shown that bacterial vaginosis (BV) is particularly prevalent in patients with infertility, though it has not been firmly established which risks infertility patients with BV incur for pregnancy outcome. We aimed to assess the prevalence of BV in infertility patients, as well as to quantify the magnitude of the association between BV and cause of infertility on the one hand, and conception rates and early pregnancy loss following in-vitrofertilisation (IVF) on the other hand.

Methods Systematic literature review and meta-analysis.

Results The estimated prevalence of BV in infertile women is $19 \%$ (95\% CI: $14-25 \%$ ). Abnormal microbiota (Nugent scores 4 to 10) occurs in $39 \%$ of the infertile patients ( $95 \%$ CI: $26-52 \%$ ). BV is significantly more prevalent in women with infertility compared to antenatal women in the same population (OR 3.32, 95\% CI $1.53-$ 7.20). BV is significantly more prevalent in women with tubal infertility compared to women with other causes of infertility (OR 2.77, $95 \%$ CI $1.62-4.75)$. BV is not associated with decreased conception rates (OR $1.03,95 \%$ CI $0.79-1.33$ ). BV is associated with a significantly elevated risk of preclinical pregnancy loss (OR 2.36, 95\% CI: $1.24-4.51)$, but is not associated with an increased risk of first trimester abortion (OR 1.20, 95\% CI: $0.52-2.74$ )

Conclusion All studies on cause of infertility in relation to BV included had a cross-sectional design and therefore do not allow for causal inferences. Still, there is strong circumstantial evidence that supports a causal link between BV and tubal infertility. Studies with a longitudinal design on other hand strongly support a relation between BV and early pregnancy loss. Unfortunately, no study looked beyond first trimester foetal loss.

\section{P1.028 TREATMENT OF RECURRENT BACTERIAL VAGINOSIS WITH OCTENIDINE DIHYDROCHLORIDE}

doi:10.1136/sextrans-2013-051184.0249

'H Verstraelen, ${ }^{2} \mathrm{~V}$ Loening-Baucke, ${ }^{3} \mathrm{~S}$ Swidsinski, ${ }^{2} \mathrm{~A}$ Swidisinski. ' Ghent University, Ghent, Belgium; 'Laboratory for Molecular Genetics, Polymicrobial Infections and Bacterial Biofilms, Berlin, Germany: ${ }^{3}$ Department of Microbiology, Labor Berlin, Berlin, Germany

Background Bacterial vaginosis is a recalcitrant polymicrobial biofilm infection that often resists standard antibiotic treatment. We therefore considered repeated treatment with octenidine, a local antiseptic that has previously been shown to be highly effective in several biofilm-associated infections.

Methods Twenty-four patients with recurrent BV were treated with a 7-day course of octenidine (octenidine dihydrochloride spray application with the commercial product Octenisept ${ }^{\circledR}$ ). In case of treatment failure or relapse within six months patients where retreated with a 28 day course of octenidine. In case of recurrence within six months after the second treatment course, patients were treated again with a 28 day course followed by weekly applications for two months. Treatment effect was evaluated by assessment of the presence of the biofilm on voided vaginal epithelial cells through fluorescence-in-situ-hybridisation (FISH).

Results The initial cure rate following a 7-day course of octenidine was as high as $87.5 \%$. The six-month relapse rate was however as high as $66.6 \%$. Repeated treatment for 28 days led to an overall cure rate of $75.0 \%$, however was also associated with emergence of complete resistance to octenidine in a subset of women. The overall cure rate after three treatment courses with one year follow-up was $62.5 \%$, with $37.5 \%$ of the patients showing complete resistance to octenidine.

Conclusion Although octenidine dihydrochloride was initially highly effective, it was also found that the efficacy of repeated and prolonged treatment dropped quickly as challenge with the antiseptic rapidly led to bacterial resistance in a considerable subset of women.

\section{P1.029 BACTERIAL VAGINOSIS ASSOCIATED BACTERIA ARE DETECTED TOGETHER WITH UREAPLASMAS IN MEN BUT NOT ASSOCIATED WITH NON-GONOCOCCAL URETHRITIS}

doi:10.1136/sextrans-2013-051184.0250

'M Frølund, 'R Datcu, 'P Ahrens, 'L Falk, 'I S Jensen. 'Statens Serum Institut, Copenhagen, Denmark; ${ }^{2}$ Dept. of Dermatovenereology, Linköping, Sweden

Background Little is known about the presence of bacterial vaginosis (BV) associated bacteria in men, but male partners of women with BV have been reported to have a high risk of urethritis. We aimed to examine the role of $\mathrm{BV}$ associated bacteria in urine specimens from men with and without non-gonococcal urethritis (NGU). Methods First-pass urines were collected from 44 men with symptomatic NGU ( $\geq 5 \mathrm{PMNL} / \mathrm{hpf}$ ) and 97 asymptomatic men without $\mathrm{NGU}(<5 \mathrm{PMNL} / \mathrm{hpf})$. Samples were tested for Chlamydia trachomatis (Ct), Mycoplasma genitalium (Mg), Ureaplasma urealyticum (Uu), U. parvum (Up), HSV 1 and 2, and adenovirus by PCR.

Quantitative PCRs were performed to detect Gardnerella vaginalis, BVAB 2, Eggerthella-like uncultured bacterium, Megasphaera type 1, Leptotrichia amnionii, Atopobium vaginae, Sneathia sanguinegens, and Prevotella sp.

Results Ct was detected in 9 (21\%) cases with NGU and $1(1 \%)$ control without NGU. Mg was detected in 10 cases (23\%) and none of the controls. Corresponding figures were for $\mathrm{Uu} 4(9 \%)$ and 26 $(27 \%)$, and Up in $6(14 \%)$ and $25(26 \%)$, respectively. HSV type 1 was found in 2 case samples (5\%). Controls were all negative for HSV. Adenovirus was found in 2 NGU samples and none of the controls. In 20 (46\%) NGU cases no aetiology was found.

Conclusion G. vaginalis, BVAB-2, Eggerthella, L. amnionii, A. vaginae, S. sanguinegens and Prevotella, but not Megasphaera type 1, had an increasing bacterial load with increasing total Ureaplasma sp. load in male urine regardless of NGU status or co-infections with known NGU pathogens. None of the BV bacteria were associated with NGU.

Correlation between total Ureaplasma sp. and BV bacterial load in all samples:

\section{Abstract P1.029 Table}

\begin{tabular}{lll}
\hline BV bacteria & Spearman-Correlation & p-value \\
\hline G. vaginalis & 0.59 & $<0.0001$ \\
BVAB-2 & 0.28 & 0.0008 \\
Eggerthella & 0.49 & $<0.0001$ \\
Megasphaera & -0.05 & 0.57 \\
L. amnionii & 0.31 & 0.0003 \\
A. vaginae & 0.36 & $<0.0001$ \\
S. sanguinegens & 0.27 & $<0.0001$ \\
Prevotella sp. & 0.14 & 0.0003 \\
\hline
\end{tabular}

